\title{
Condições de trabalho e os impactos na saúde dos motoristas de transporte de lotação
}

\author{
Working conditions and health impacts of stock transport drivers
}

\author{
Izete Soares da Silva Dantas Pereira* \\ Ana Jéssica Nunes Araujo**
}

\begin{abstract}
Resumo
O trabalho realizado pelos motoristas de transporte de lotação se caracteriza por condições precárias com repercussões à saúde. A jornada de trabalho com consequente sobrecarga física e psicológica provoca o aparecimento de sintomas e sinais físicos e mentais, doenças crônicas e agudas. O objetivo geral da pesquisa foi conhecer as Condições de trabalho e os impactos na saúde dos motoristas de transporte de lotação. Tratou-se de estudo transversal descritivo e exploratório com abordagem qualitativa a partir da realização de entrevistas semiestruturadas com 34 profissionais associados de uma Cooperativa de Transportes de Lotação no Rio Grande do Norte. Os resultados mostraram que as condições de trabalho dos motoristas se caracterizam por excesso de horas, doenças cardiovasculares, psicofisiológicas, ausência de cuidados preventivos, além de outros problemas que impactam na saúde. Há necessidade de maior investimento em ações de prevenção e promoção da melhoria da saúde desses profissionais a fim de que sejam asseguradas melhores condições de trabalho minimizando os fatores de risco a que estão expostos.
\end{abstract}

Palavras-chave: Condições de trabalho, Motoristas de Transportes de lotação, Impactos na saúde, Fatores de risco para a saúde, Prevenção e promoção da saúde.

\section{Abstract}

The work carried out by the drivers of alternative transportation service is characterized by precarious conditions with repercussions to health. The workday with consequent physical and psychological overload causes the appearance of physical and mental symptoms and signs, chronic and acute diseases. The general objective of the research was to know the Working Conditions and the impacts on the health of the drivers of the transportation of the stocking. This was a descriptive and exploratory cross-sectional study with a qualitative approach based on the performance of semi-structured interviews with 34 associated professionals of a Cooperation of alternative transportation service in Rio Grande do Norte. The results showed that drivers' working conditions are characterized by excessive hours, cardiovascular and psychophysiological diseases, lack of preventive care, and other problems that impact health. There is a need for greater investment in actions to prevent and promote the improvement of the health of these professionals in order to ensure better working conditions, minimizing the risk factors to which they are exposed.

Keywords: Working conditions, driver of alternative transportation service, Impacts on health, Health risk factors, prevention and health promotion.

\footnotetext{
* Docente do Curso de Serviço Social do Centro Universitário do Rio Grande do Norte -UNIRN. Docente do Curso de Medicina da Universidade Estadual do Rio Grande do Norte - UERN

** Assistente Social pelo Centro Universitário do Rio Grande do Norte-UNIRN.
} 


\section{Introdução}

Desde os primórdios que há registros da relação entre os processos de trabalho e sua influência sobre a saúde e o adoecimento das populações. O ser humano trabalha para sua sobrevivência, recebendo um salário, na maioria das vezes, insuficiente para a sua subsistência e de sua família. A OIT (Organização Internacional do Trabalho) e a OMS (Organização Mundial da Saúde) têm destacado o estreito relacionamento existente entre o trabalho e a saúde. A evolução no conceito de saúde mostra que a partir do século XIX, a atenção era dirigida quase exclusivamente para a doença, as mortes, os acidentes de trabalho e as doenças profissionais que ceifava a vida dos trabalhadores.

As condições de trabalho a que a população, normalmente, se encontra exposta, camuflam as expressões de outras necessidades, como as de lazer e de satisfação pessoal. Nessa perspectiva, o trabalho aparece afastado do prazer, contribuindo para que o ser humano, nem sempre, consiga encontrar, nas horas vagas, meios de satisfação que compense o trabalho exaustivo (MOURA NETO; SILVA, 2012; NEFFA, 2015).

No contexto da sociedade capitalista, o trabalho - atividade central na vida do ser humano - é extremamente valorizado. No entanto, nem todas as suas dimensões são explicitadas e discutidas. Observa-se uma certa resistência social em reconhecer que o seu exercício pode resultar em sentimentos de satisfação, realização e prazer, mas também pode ter como consequência mal-estar, doenças e acidentes (CARDOSO; MORGADO, 2019; ASSUNÇÃO; FRANÇA, 2020).

A Constituição da República Federativa do Brasil de 1988 afirma em seu capítulo II, dos direitos sociais no artigo 6으, que são direitos "a educação, a saúde, a alimentação, o trabalho, a moradia, o transporte, o lazer, a segurança, a previdência social, a proteção à maternidade e a infância, a assistência aos desamparados" [...] (BRASIL, 2018, p. 8).

Da mesma forma, o texto constitucional na seção II da saúde em seu artigo 196 afirma que:

A saúde é um direito de todos e dever do Estado, garantido mediante políticas sociais e econômicas que visem à redução do risco de doenças e de outros agravos e ao acesso universal e igualitário às ações e serviços para sua promoção, proteção e recuperação (BRASIL, 2018, p. 55). 
O trabalho é uma atividade que responde às necessidades e carências do indivíduo, sendo, essencialmente humana. Portanto, deve possibilitar a autotransformação do trabalhador devendo ser uma prática livre e autônoma, levando à emancipação e humanização. As mudanças ocorridas na trajetória histórica do processo de trabalho mostram que, inicialmente era visto como atividade penosa e árdua e que, posteriormente, passou a ocupar centralidade na vida de todos os homens como forma de direito a ser assegurado constitucionalmente (NEFFA, 2015; ANTUNES, 2013).

O trabalho, nos dias atuais, tem sido considerado um importante fator gerador de estresse, sendo um dos problemas que mais frequentemente agem sobre o ser humano e interfere no organismo, devido à grande quantidade de tensões que provoca diariamente. Estudos realizados sobre as condições de trabalho têm mostrado que hábitos e estilos de vida - sedentarismo, alimentação inadequada, poucas horas de sono, tabagismo, estresse, etc. interferem na exposição desses profissionais aos riscos cardiovasculares, mas também, de outras doenças (SILVA; ASSUNÇÃO; NEVES, 2013; NEVES; MACEDO; GOMES, 2017).

Condições gerais de vida, de trabalho, ambiente de trabalho são fatores que, como outros determinam situações diversas na vida dos motoristas que podem influir negativamente na sua saúde (RAMOS; ALMEIDA; RIBEIRO; SILVEIRA, 2018; ALCANTARA, 2016; COSTA; COSTA; CINTRA, 2018; CARDOSO; MORGADO, 2019).

No contexto atual, as formas de gestão, as condições organizacionais e físicas do trabalho, as relações de trabalho e as situações e estão determinando e contribuindo para o adoecimento dos trabalhadores (CARDOSO, 2015).

Vários estudos, brasileiros e estrangeiros, têm demostrado que a profissão de motorista apresenta um adoecer e morrer diferenciado da população em geral (SANTOS JÚNIOR, 2003; BRITO; GOIS; ALMEIDA; MARTINS; RODRIGUES; GOIS JUNIOR, 2016; FERREIRA; GRAMS; ERTHAL; GIRIANELLI; OLIVEIRA, 2018; CARDOSO; MORGADO， 2019; ASSUNCAO; FRANCA, 2020).

Santos Júnior realizou uma revisão da literatura que abrangeu um período de 15 anos sobre morbimortalidade de motoristas de ônibus. Ficou constatado que os motoristas estão expostos a uma diversidade de fatores nocivos (físicos, químicos, biológicos e ergonômicos). Esses fatores podem produzir doenças, relacionadas ao trabalho em várias categorias. exercício da profissão de motorista nas condições de trabalho em que estão, normalmente 
submetidos em praticamente todo o mundo, afeta de forma direta e indireta a saúde desses trabalhadores, causando formas variadas de sofrimento físico e/ou mental, adoecimento e mortes prematuras (2002).

A atividade realizada pelos motoristas profissionais se caracteriza, na maioria das vezes, por uma rotina com as consequentes repercussões à saúde. Inclui muitas horas ao volante, longe das famílias e de seu ambiente social com turnos irregulares. Além disso, a falta de uma infraestrutura adequada, resultado de vários anos de baixos investimentos públicos, que restringem o desenvolvimento do país e das empresas, afeta diretamente a segurança e a qualidade de vida desses trabalhadores (ALCANTARA et al, 2016; CARDOSO et al, 2015; FERREIRA; ALVAREZ, 2013).

Ressalta-se que a vida no ambiente de trabalho e fora dele, ou seja, nos espaços da produção e do consumo estão de tal forma interligados que não é possível separá-los e, assim, eles se auto influenciam. As condições nas quais o trabalho é realizado podem atuar tanto na melhoria das habilidades humanas- oferecendo melhor qualidade de vida e saúde quanto provocando o adoecer, o agravamento do adoecer e até mesmo diminuindo os anos potenciais de vida dos trabalhadores (BRASIL, 2016).

Os agravos à saúde desses trabalhadores estão imbricados com diversos tipos de risco, como já mencionados. Os trabalhadores ficam sujeitos à exposição a aspectos materiais, físicos, químicos, biológicos, culturais e organizacionais nos processos de trabalho. Nesse sentido, uma abordagem integrada do ambiente de trabalho, propiciaria ações menos fragmentadas, servindo de base para legislações e políticas públicas que respeitem a realidade e a dignidade do trabalhador, em geral e desse, em particular (FERREIRA; GRAMS; ERTHAL; GIRIANELLI; OLIVEIRA, 2018).

A partir do conhecimento de que as atividades dos motoristas de transporte podem causar danos à saúde e à integridade física, psíquica e social devido à sua natureza, suscetibilidade, intensidade, tempo de exposição, entre outros fatores definiu-se como objetivo conhecer as Condições de trabalho e os impactos na saúde dos motoristas de transporte de lotação de uma Cooperativa no estado do Rio Grande do Norte.

No mundo contemporâneo, o processo de viver humano é marcado pelo crescimento das incertezas e da sensação de fragilidade diante dos fatores de risco e vulnerabilidade aos quais todas as pessoas, direta ou indiretamente, estão expostas. Essas características 
repercutem também no cotidiano de trabalho dos indivíduos, pois o trabalho é um dos principais eixos estruturantes da vida humana. No âmbito das práticas laborais dos profissionais da saúde, as questões referentes a risco e/ou vulnerabilidade estão ainda mais presentes, uma vez que esses profissionais se expõem rotineiramente a múltiplos e variados riscos relacionados a agentes químicos, físicos, biológicos e psicossociais (FERREIRA; GRAMS; ERTHAL; GIRIANELLI; OLIVEIRA, 2018).

Diversos interesses envolvidos, tem levado a sociedade a ocultar o adoecimento no trabalho e muitas vezes a culpabilizar e responsabilizar o trabalhador pela "sua" doença. Para que as dimensões da satisfação, da realização e do prazer no trabalho possam prevalecer, é necessário trazer para o centro do debate os determinantes sociais do mal-estar no trabalho, buscando compreender de quais sofrimentos falam os trabalhadores1 - isto é, conhecer como o cotidiano do trabalho, que passa por forte processo de precarização, flexibilização, intensificação, crescente desemprego e insegurança (CARDOSO, 2014; NEFFA, 2015) tem influenciado a vida e a saúde do trabalhador. Para entender como a atividade de trabalho é realizada, torna-se fundamental conhecer de forma detalhada a gestão, a organização, as condições e as relações de trabalho em cada setor, em cada célula, em cada posto de trabalho - conhecer como os indivíduos enfrentam os riscos, as doenças e os acidentes relacionados ao trabalho.

\section{Impactos do trabalho na saúde}

A saúde tem sido entendida como um conceito ampliado, resultante das condições de alimentação, habitação, educação, renda, meio-ambiente, trabalho, transporte, lazer, liberdade, acesso à posse de terra e a serviços de saúde desde a VIII Conferência Nacional de Saúde realizada em 1986 (TORRES, 2020).

A partir desse entendimento há um consenso entre a maior parte dos estudiosos que há fatores de risco (físicos, químicos, biológicos, psicossociais) decorrentes das condições de trabalho que interferem no estado psicofisiológico, traduzindo-se em irritabilidade, insônia, distúrbios da atenção e em sintomas osteomusculares, além de outros. Esses últimos destacam-se em diversos estudos que descrevem a prevalência associada à sintomatologia desses problemas em diferentes ocupações, incluindo a de motoristas (FERREIRA; GRAMS; 
ERTHAL; GIRIANELLI; OLIVEIRA, 2018). Alguns problemas de saúde ou de possibilidades de adoecimento - obesidade, hipertensão, taxas alteradas de colesterol, diabetes, alterações posturais - acentuadas pela situação de trabalho dos motoristas profissionais levando-se em consideração aspectos relacionados à organização do trabalho (DE VITTA, 2013; ASSUNÇÃO; FRANÇA, 2020; FERREIRA; GRAMS; ERTHAL; GIRIANELLI, OLIVEIRA, 2018; FERREIRA; ALVAREZ, 2013).

Os riscos no ambiente laboral podem causar danos à saúde e à integridade física do trabalhador devido à sua natureza, suscetibilidade, intensidade, tempo de exposição e concentração. Podem ser elencados, no caso da profissão de motoristas os riscos físicos como ruído excessivo, temperatura inadequada, má qualidade do ar que respiram, além de outros. Já os químicos se dão pela exposição - as principais cargas químicas mencionadas são relacionados aos produtos que transportam e que estão em contato a depender da atividade que desenvolvem, por exemplo os agrotóxicos, medicamentos, solventes e desinfetantes, combustíveis. Os biológicos, estão relacionados aos profissionais que atuam na área da saúde e trabalham em veículos que transportam pacientes, com diferentes problemas de saúde, incluindo as doenças infecto-contagiosas. Os agravos à saúde desses trabalhadores apresentam-se imbricados com diversos tipos de riscos, como já mencionados. Os motoristas ficam sujeitos à exposição a aspectos materiais, físicos, químicos, biológicos, culturais e organizacionais nos processos de trabalho. Uma abordagem integrada do ambiente de trabalho, nesse sentido, propiciaria ações menos fragmentadas, servindo de base para legislações e políticas públicas que respeitem a realidade e a dignidade do trabalhador, em geral e desse, em particular (FERREIRA; GRAMS; ERTHAL; GIRIANELLI; OLIVEIRA, 2018).

A partir de estudos realizados, nessa área, foram encontrados vários problemas para a saúde desse trabalhador, entre eles: doenças cardiovasculares, osteomusculares, altas taxas de sedentarismo, obesidade, hábitos alimentares inadequados, estresse, longas jornadas de trabalho, poucas horas de sono, insônia, uso de drogas estimulantes para a manutenção da vigília, vulnerabilidade às práticas de risco para as DST/AIDS, entre outros (ALCANTARA, 2016; LOPES et al, 2012).

A influência da jornada de trabalho com horários irregulares e a consequente sobrecarga física e psicológica evidencia-se no aparecimento de sintomas e sinais físicos e mentais, tais como problemas de relacionamento, solidão, sonolência, irritabilidade, fadiga, 
reflexos diminuídos, que podem levar ao aparecimento tanto de doenças crônicas quanto de agudas, entre estas os politraumatismos por acidentes nas rodovias (KNAUTH, 2012; GORDIA et al, 2011).

O excesso de trabalho, como mostram vários estudos realizados com motoristas profissionais é uma das maiores queixas. Os motoristas afirmam que essas jornadas longas e o estresse Ihes afeta a qualidade de vida. Referem-se a jornadas intensas que exigem mais tempo, mais rapidez, mais atenção ou, ainda, consistem em tarefas complexas, implicando, mais provavelmente, na exaustão física por sobrecarga de atividade.

Esses fatores de risco decorrentes das condições de trabalho interferem no estado psicofisiológico, traduzindo-se em irritabilidade, estresse, insônia, distúrbios da atenção e em sintomas musculoesqueléticos, ou seja, provocando doenças ocupacionais em cerca de $71 \%$ dos motoristas entrevistados, neste estudo.

Nesse sentido, estudiosos alertam para o custo psíquico (cognitivo e afetivo) imposto aos trabalhadores em sua integridade biopsíquica e física e refletem sobre a realização do trabalho e a qualidade de vida, com longas jornadas, durante dias seguidos, no cumprimento das suas tarefas sob condições-limite: vigília-sono (FERREIRA; ALVAREZ, 2013; CARDOSO; MORGADO, 2019; ASSUNÇÃO; SILVA, 2013).

A principal fonte de queixa é o excesso de trabalho, ou seja, jornadas intensas que exigem mais tempo, mais rapidez, mais atenção, levando à exaustão por sobrecarga. O ambiente de trabalho dos profissionais que transportam pessoas apresenta aspectos negativos e conflitantes como os congestionamentos, a precariedade dos veículos e a violência urbana. Os motoristas lidam com vários fatores ao mesmo tempo, gerando estresse. A complexidade, a intensidade e a exaustão, o excesso de trabalho se constitui uma fonte de desgaste (CARDOSO, 2015; ALCANTARA, 2016).

\section{Metodologia}

Tratou-se de um estudo transversal descritivo e exploratório de abordagem qualitativa com motoristas de lotação associados de uma Cooperativa de Transportes de Lotação de longa duração que atuam no Rio grande do Norte/Brasil no período de maio de 2015 a março de 2016. 
Foi considerado, para a pesquisa, o universo dos associados. No momento da coleta, havia sessenta (60) motoristas cadastrados na Cooperativa. No entanto, por razões diversas participaram das entrevistas 34 motoristas $(56,7 \%)$, após a assinatura do Termo de Consentimento Livre e Esclarecido-TCLE. Um dos critérios para encerramento da pesquisa fora a participação de, pelo menos, $50 \%$ do universo.

Os critérios de inclusão foram: ser associado da cooperativa, desenvolver a atividade de motorista de forma profissional, estar em plena atividade laboral.

Foram excluídos os colaboradores eventuais sem vínculos com a Cooperativa, os que estiveram ausentes da cooperativa durante o período da coleta de dados, os que se encontravam afastados do trabalho por doença e/ou problemas pessoais.

Muitos alegaram falta de tempo para conceder a entrevista uma vez que a atividade por eles desenvolvida não tem horário de início ou fim, previamente determinado. Trabalham na medida em que existe demanda, podendo essa carga horária exceder as 8 horas exigida por Lei.

A coleta dos dados deu-se a partir da realização de uma entrevista estruturada, utilizando como instrumento um roteiro com perguntas fechadas e abertas. Os dados foram coletados na sede da Cooperativa, de forma individual, em sala reservada, respeitando as Normas da Resolução 466/12 do Conselho Nacional de Saúde - CNS e suas complementares.

O Roteiro para a realização da entrevista consistiu de itens sobre variáveis sócio demográficas, como faixa etária (em anos), escolaridade (ensino fundamental, médio e superior), estado civil (solteiro, casado, viúvo, em união estável) tempo de profissão (em anos). Em relação às condições de trabalho questionou-se a jornada de trabalho diária número de horas trabalhadas em média durante o dia, tempo de profissão, conhecimento da Lei que disciplina a profissão de motorista. Foi considerado pertinente ao estudo a identificação dos hábitos do tabagismo e da ingestão de bebidas alcoólicas a pratica de algum tipo de esporte, atividades de lazer e se faz exames preventivos de rotina para preservar a saúde, o uso atual ou pregresso de medicamentos sem prescrição médica, seja, automedicação. Constou ainda, do roteiro, o conhecimento de problemas que afetam a saúde desses profissionais e o que poderia ser feito para melhorar a sua qualidade de vida, na opinião dos participantes.

A pesquisa foi aprovada pelo Comitê de Ética em Pesquisa - CEP da Universidade do Estado do Rio grande do Norte-UERN sob o no CAAE 38715514.8.0000.5294. 
Os dados sociodemográficos foram apresentados sob a forma de Tabela e alguns depoimentos foram transcritos, literalmente, a fim de manter a fidedignidade da informação.

\section{Resultados}

Os resultados das entrevistas realizadas podem ser visualizados na Tabela a seguir. Foram entrevistados 34 motoristas que trabalham com transporte de lotação.

Tabela 1. Dados sociodemográficos e de saúde dos entrevistados-Mossoró, 2017

\begin{tabular}{|c|c|c|}
\hline Faixa etária & Quantidade & $\%$ \\
\hline De 25 a 35 anos & 8 & 23,5 \\
\hline De 35 a 45 anos & 6 & 17,6 \\
\hline De 45 a 55 anos & 20 & 58,9 \\
\hline \multicolumn{3}{|l|}{ Estado civil } \\
\hline Solteiro & 2 & 5,7 \\
\hline Casado & 26 & 76,5 \\
\hline União estável & 4 & 11,9 \\
\hline Separado & 2 & 5,9 \\
\hline \multicolumn{3}{|l|}{ Escolaridade } \\
\hline Ensino fundamental & 18 & 52,9 \\
\hline Ensino médio & 14 & 41,2 \\
\hline Ensino Superior & 2 & 5,9 \\
\hline \multicolumn{3}{|l|}{ Tempo de profissão } \\
\hline De 1 a 5 anos & 4 & 11,7 \\
\hline De 5 a 10 anos & 6 & 17,6 \\
\hline Mais de 10 anos & 24 & 70,7 \\
\hline \multicolumn{3}{|l|}{$\begin{array}{l}\text { Jornada média de } \\
\text { trabalho diária }\end{array}$} \\
\hline Até 8 horas & 14 & 41,0 \\
\hline De 8 a 10 horas & 14 & 41,0 \\
\hline Acima de 10 horas & 6 & 18,0 \\
\hline \multicolumn{3}{|l|}{ Hábitos } \\
\hline Fuma & 4 & 11,7 \\
\hline Bebe & 23 & 67,3 \\
\hline Pratica atividade física & 5 & 14,7 \\
\hline Lazer & 6 & 18,0 \\
\hline Automedicação & 14 & 41,0 \\
\hline \multicolumn{3}{|l|}{ Problemas de saúde } \\
\hline Hipertensão & 20 & 59,0 \\
\hline Diabetes & 12 & 53,0 \\
\hline Estresse & 12 & 53,0 \\
\hline Fadiga & 18 & 35,0 \\
\hline
\end{tabular}




\begin{tabular}{l|c|c}
\hline Insônia & $\mathbf{1 7}$ & $\mathbf{5 0 , 0}$ \\
\hline Gastrointestinais & $\mathbf{8}$ & $\mathbf{2 3 , 5}$ \\
\hline Osteomusculares & 15 & 44,0 \\
\hline Outros & 12 & 53,0 \\
\hline
\end{tabular}

Fonte: Elaborado pelas autoras

\section{Discussão}

Todos os motoristas entrevistados eram do sexo masculino, sendo que a faixa etária predominante foi entre 45 e 55 anos de idade, representando 58,8\%. Quanto ao estado civil, os casados são maioria com 76,5\%. Dessa forma é possível observar na tabela que somados aqueles que têm uma união estável, têm-se $88,0 \%$. A maioria, 35,3\%, possui apenas o ensino fundamental completo, seguidos por $29,4 \%$ com o ensino médio. Apenas 5,9\% possuem curso superior completo. Dessa forma mais de $60,0 \%$ não chegaram a concluir o ensino médio, o que mostra uma baixa escolaridade no grupo.

Um aspecto considerado importante foi conhecer o tempo de experiência profissional dos participantes. Questionados, 70,7\% relataram mais de 10 anos de exercício profissional. Quanto aos motivos da escolha da profissão foram citados falta de oportunidade $35,3 \%$, desejo pessoal $29,4 \%$, influência familiar $17,7 \%$ e outros $17,6 \%$. Alguns participantes afirmaram que não tiveram oportunidade para o exercício de outra atividade, pois não puderam frequentar uma Faculdade e fazer um curso superior ou mesmo profissionalizante. Outros disseram que casaram muito cedo e logo vieram os filhos, tendo que trabalhar no que aparecesse para manter a família. A baixa escolaridade fez com que fossem desempenhar uma atividade prática, pois já sabiam dirigir e assim tiveram só que tratar da documentação e começar a trabalhar. Não tiveram oportunidades de fazer outras escolhas na vida e aproveitaram o que a vida ofereceu, pois o pai já era motorista e exercia a mesma atividade, sendo mais fácil continuar a profissão do pai.

A informação sobre a jornada de trabalho diária dos entrevistados mostrou que $58,8 \%$ dirigem acima de 10 horas e, às vezes, até 14 horas. Alguns desses motoristas chegam a trabalhar quase o dobro do permitido por Lei (BRASIL, 2016). A rotina de trabalho e a necessidade os obriga a jornadas longas para manter os compromissos financeiros para si e/ou para a manutenção da família. Apesar das dificuldades e da sobrecarga de horas trabalhadas a maioria, $(72,8)$ afirmou estar satisfeita com a profissão. 
A esse respeito questionou-se conhecem a lei 12.619, que trata do exercício da profissão de motorista, disciplinando o número de horas trabalhadas por dia. Somente $29,41 \%$ dos entrevistados disseram sim. A maioria, 70,59\% não conhece a Lei criada para limitar a carga horária dos motoristas. A Lei número 12.619 de 30 de abril de 2012 trouxe direitos e deveres para os motoristas profissionais, com intuito de melhorar a qualidade de vida e do exercício da profissão (BRASIL, 2016).

Vale destacar o Capitulo III-A Da condução de veículos por motoristas profissionais. O artigo 67 afirma que é vedado ao motorista profissional, no exercício de sua profissão e na condução de veículo mencionado no inciso II do art. 105 deste Código, dirigir por mais de 4 (quatro) horas ininterruptas. A questão que o $\S 1$ ㅇ do Art.167 traz, é um assunto de extrema importância para esses profissionais, pois exige um intervalo mínimo de 30 (trinta) minutos para descanso a cada 4 (quatro) horas ininterruptas na condução de veículo referido no caput, sendo facultado o fracionamento do tempo de direção e do intervalo de descanso, desde que não completadas 4 (quatro) horas contínuas no exercício da condução.

Os estilos de vida foram indagados a partir da identificação do uso de drogas lícitas e/ou ilícitas, uso de automedicação, pratica de esporte e lazer. Neste estudo, a ingestão de bebidas alcoólicas foi declarada por $67,3 \%$ dos entrevistados, dos quais $45,0 \%$ relataram o consumo de, pelo menos, uma vez por semana. O tabagismo mostrou uma menor prevalência com $11,7 \%$. Quanto ao uso de medicamentos sem receita (automedicação) 41,0\% declararam usar algum analgésico para as dores, um relaxante muscular ou medicamento para o estomago. A prática de alguma atividade física foi relatada por apenas $12,7 \%$ e esta se limita “a jogar futebol com os colegas nos fins de semana, quando não trabalham ou não tem compromissos familiares". O lazer desse grupo é "ver televisão, visitar amigos e familiares", mas raramente $(18,0 \%)$. Como pode ser observado os participantes possuem hábitos pouco saudáveis considerando a atividade que desenvolvem. Constata-se que a maior parte dos comportamentos estão relacionados com as condições de trabalho e com os estilos de vida desses profissionais (ALVARES et al, 2018).

Com o objetivo de analisar a incidência de uso de álcool e drogas, um estudo com 91 caminhoneiros mostrou que $66 \%$ dos participantes usavam anfetaminas durante as viagens, adquirida, principalmente, em postos de combustíveis (54\%) à beira das rodovias. 0 álcool era utilizado por $91 \%$ deles, dos quais $43 \%$ também consumiam bebidas alcoólicas. Os autores 
concluíram que há a necessidade de campanhas preventivas e informativas voltadas para essa categoria profissional, alertando sobre os riscos de ingestão dessas substâncias no período de trabalho (MASSON; MONTEIRO, 2010).

Outros autores também constataram em suas pesquisas o consumo de algum tipo de estimulante de forma isolada ou em combinação com outras substâncias (café, guaraná em pó, energéticos, cocaína aspirada) para diminuir o cansaço, evitar o sono e se manter acordado. Estudo conduzido por Puente-Rodriguez e Pillon (2011) constataram resultados semelhante no qual mais da metade dos motoristas entrevistados em sua pesquisa faziam uso de bebidas alcoólicas e referiram já ter usado substâncias inibidoras do sono, sendo que 19\% eram tabagistas. O uso do rebite foi a droga mais citada e esteve associado às faixas etárias mais jovens entre os motoristas (PUENTE-RODRIGUEZ; PILLON, 2011; SANTOS; RODRIGUES; VENANCIO; BRAGANÇA E SILVA; FERREIRA; FERNANDES, 2017).

Para evitar a sonolência, muitos motoristas consomem estimulantes sob a forma de anfetaminas ou outras drogas. A esse respeito vale citar um estudo realizado com os caminhoneiros que trafegam na BR 153 com o objetivo de conhecer o uso de drogas e outras substâncias psicoativas consumidas por esses profissionais. O uso de anfetaminas (rebite) foi informado por $32,9 \%$ dos caminhoneiros que afirmaram ter feito uso dessa droga nos últimos três meses. Relataram, ainda, não encontrar dificuldades para adquirir essas drogas, sendo os postos de gasolina o local mais citado de acordo com $38,3 \%$ dos participantes. Isso reflete a falta de fiscalização por parte dos órgãos responsáveis e de conscientização destes profissionais uma vez que o consumo dessas substâncias é um dos fatores de risco para acidentes rodoviários (SANTOS; RODRIGUES; VENANCIO; BRAGANÇA E SILVA; FERREIRA; FERNANDES, 2017).

Outros problemas que também são considerados fatores de risco são: distúrbios do sono não tratados ou não diagnosticados; perda de sono; horários e pausas inapropriadas e consumo de álcool (KUBOTA et al, 2014; NEVES, MACEDO; GOMES, 2017; CAMARGO et al, 2013).

A realização de exames preventivos foi um dos aspectos questionados aos motoristas. Mais da metade, $58,8 \%$, afirmaram que não os realizam. Buscam os serviços de saúde apenas quando estão doentes ao ponto desse agravo impossibilitá-los para o trabalho. Apenas $23,5 \%$ disseram que fazem exames de rotina de forma preventiva e $17,6 \%$ declararam 
que às vezes os fazem, quando se faz necessário, por exemplo para a renovação da Carteira de Habilitação. A ausência de sinais e sintomas, na maioria das vezes, dificulta a percepção dos riscos a que estão expostos ou mesmo a existência de alguma doença em curso. 0 desencadeamento das diversas condições crônicas de saúde é bem documentado na literatura quando se refere a esse grupo de profissionais (SANTOS JUNIOR, 2002; RAMOS; ALMEIDA; RIBEIRO; SILVEIRA, 2018; FERREIRA; ALVAREZ, 2013; OTONNI; CARLOS, 2012).

A existência de problemas de saúde, por eles conhecidos e que lhes afeta as atividades laborais e a qualidade de vida foi um dos itens questionados.

Dentre os problemas de saúde mais referidos pelo grupo pesquisado se encontram insônia, (58,8\%), hipertensão (50\%), (fadiga 29,4\%), dores osteomusculares $(18,0 \%)$ diabetes $(12,0 \%)$, gastrointestinais $(11,8 \%)$, ansiedade $(11,8 \%)$, além de outros. Vários problemas foram citados de forma simultânea ultrapassando, cem por cento. Esses problemas são gerados, principalmente pelo tipo de atividade que desenvolvem e pela qualidade de vida a que estão expostos, na opinião da maioria (94,11\%).

Kubota et al (2014) apontam que as perturbações do sono podem provocar alterações significativas no funcionamento físico, ocupacional, cognitivo e social do indivíduo, além de interferir na qualidade de vida. Nesse sentido, a insônia é um dos distúrbios do sono, que pode implicar negativamente no desempenho individual afetando a saúde das pessoas. Aspectos relacionados, principalmente, com a memória; concentração e atenção; fadiga; menor produtividade; absenteísmo, distúrbios de humor; ansiedade; depressão; fadiga; ansiedade e estresse. Alguns desses problemas foram citados pelo grupo de motoristas que fizeram parte deste estudo.

Em vários estudos realizados, a hipertensão arterial apresenta associação significativa com os maiores níveis de índice de massa corporal, circunferência abdominal, glicemia e triglicerídeos, além da diabetes. No contexto das doenças crônicas a hipertensão arterial, obesidade e diabetes mellitus, apresentam características similares: são crônicas, devem ser tratados por toda a vida e, muitas vezes, não apresentam sinais específicos. 0 controle da hipertensão e do excesso de peso corporal tem em vista a prevenção de complicações, além de favorecer o controle de outras morbidades associadas, evitando dessa forma a mortalidade precoce. Autores afirmam que, apesar de a hipertensão atingir pessoas de diferentes faixas etárias e em ambos os sexos, há maior prevalência desse agravo em 
pessoas que desempenham determinadas atividades de trabalho. Relaciona-se isso a fatores como estresse, sobrecarga de trabalho e responsabilidades, elevação da intensidade do trabalho, repetitividade, nível de concentração requerido, trabalho em turnos, além de outros, sendo mais prevalente entre os trabalhadores dos setores secundários e terciários da economia (SANTOS JUNIOR, 2002; BRITO; GOIS; ALMEIDA; MARTINS; RODRIGUES; GOIS JUNIOR, 2016; CARDOSO; MORGADO, 2019; ASSUNÇÃO; FRANÇA, 2020).

Nesse contexto, encontram-se os motoristas e os cobradores de transporte coletivo urbano. Esses trabalhadores assumem papel de destaque no cenário da saúde ocupacional, uma vez que estão expostos aos diferentes fatores de risco. Santos Junior (2002) em seu estudo sobre a saúde de motoristas de ônibus, mostrou que esse grupo apresenta alta morbidade e mortalidade, principalmente, por doenças do sistema cardiovascular, com destaque para a hipertensão.

Um estudo realizado com motoristas profissionais para verificar a prevalência da hipertensão arterial e obesidade entre motoristas, mostrou que essas pessoas podem estar mais expostas aos riscos cardiovasculares devido a características peculiares de sua profissão (SOUZA; SILVA; SILVA; PINTO, 2017).

Um estudo transversal conduzido com 460 motoristas de caminhão de uma transportadora de cargas das regiões Sul e Sudeste do Brasil, no qual os trabalhadores informaram dados sociodemográficos, estilos de vida e condições de trabalho, mostrou os seguintes resultados: A alta demanda no trabalho, o baixo apoio social e a jornada extensa diária foram referidos pelos motoristas como associados aos distúrbios psíquicos. $\mathrm{O}$ trabalho em jornadas extensas foi associado à ocorrência de distúrbios psíquicos, tanto na análise das condições gerais de trabalho quanto como fator referido como estressor pelos motoristas (ASSUNÇÃO; SILVA, 2013).

Neste estudo os problemas osteomusculares relativos à postura sentada foram referidos por $44,0 \%$. Esse tipo de postura gera alterações nas estruturas musculoesqueléticas da coluna lombar. Dados semelhantes foram encontrados em um estudo realizado por De Vitta (2013), no qual verificou que $65,7 \%$ dos trabalhadores relataram algum tipo de sintoma osteomuscular nos últimos 12 meses, localizados, principalmente, na região lombar (17,0\%), ombros e joelhos (13,3\%). Observou-se, ainda, associação entre os sintomas e o baixo nível de escolaridade, a alta demanda psicológica, o sedentarismo e o baixo nível de capacidade 
para o trabalho. Destacam-se como principais causas de mortalidade e morbidade três grupos de doenças: doenças cardiovasculares, do aparelho gastrointestinal e problemas musculoesqueléticos. Outros estudos em que se abordam dados epidemiológicos mundiais mostram a alta prevalência de dor lombar em motoristas, considerando essa profissão com maior risco de desenvolver doenças cardíacas e musculoesqueléticas (SOUZA; SILVA; SILVA; PINTO,2017; MASCARENHAS et al, 2014; SANTOS JÚNIOR; 2002).

Essas doenças são agravadas pelas condições de trabalho já que têm relação direta com o estresse, a postura, a constante tensão nas estradas e outros aspectos são de reconhecida associação com a qualidade de vida. A regulamentação da jornada de trabalho com limitação de horas diárias é, portanto, uma medida que pode auxiliar na redução do desenvolvimento de diversas patologias (BRASIL, 2016).

Os fatores que influenciam na sua saúde dentre as respostas dos entrevistados foram: stress, falta de lazer, sedentarismo, má alimentação, ambiente de trabalho e solidão, o que confirma estudos realizados por vários autores (RAMOS; ALMEIDA; RIBEIRO, 2018; SANTOS JUNIOR, 2002).

De forma geral, os motoristas se alimentam em restaurantes que, na maior parte, ofertam alimentos de alto valor calórico e baixo valor nutritivo. A associação da alimentação inadequada com o sedentarismo deixa esses profissionais mais expostos a desenvolver obesidade, problemas gastrointestinais e suas consequências, principalmente, a hipertensão (GIROTTO; LOCH; MESAS; GONZALEZ; GUIDONI; ANDRADE, 2020).

Um estudo transversal foi realizado no Porto de Paranaguá com motoristas de caminhão no sentido de identificar fatores associados aos comportamentos alimentares de risco à saúde. Entre os entrevistados 53,1\% apresentaram quatro ou mais comportamentos alimentares de risco. Tal condição esteve associada com idade inferior a 40 anos, capacidade para exercer a profissão referida como moderada/baixa/muito baixa não praticar atividade física no tempo livre, qualidade da alimentação referida como ruim/muito ruim e índice de massa corporal (IMC) alto. Mais da metade dos motoristas de caminhão apresentaram comportamentos alimentares de risco à saúde, reforçando a necessidade de estratégias de incentivo à redução desses hábitos (GIROTTO; LOCH; MESAS; GONZALEZ; GUIDONI; ANDRADE, 2020). 
A vida, no ambiente de trabalho e fora dele, ou seja, os espaços da produção e do consumo estão de tal forma interligados que não é possível separá-los e, assim, eles se auto influenciam. As condições nas quais o trabalho é realizado podem atuar tanto na melhoria das habilidades humanas- oferecendo melhor qualidade de vida e saúde- mas também provocando o adoecimento, o agravamento da saúde adoecer, podendo, até mesmo diminuindo os anos potenciais de vida dos trabalhadores. Os motoristas se encontram expostos as mais distintas situações de risco através das condições inadequadas de trabalho (SANTOS JUNIOR, 2002; CARDOSO; MORGADO, 2019).

As longas jornadas de trabalho, como foi citado pelos participantes os expõem a riscos que interferem num estilo de vida saudável, tornando-os vulneráveis a diversas práticas nocivas à sua saúde. Em geral, assim como os motoristas de caminhão, esses trabalhadores não tem tempo para atividades de lazer, o que também faz com que desenvolva problemas de saúde. Os principais problemas desencadeados pelo trabalho dos motoristas de ônibus urbano são: estresse, ansiedade, problemas gastrointestinais, pressão alta, angústia, problemas osteomusculares, obesidade, visão irritada, agressividade, problemas respiratórios, problemas auditivos, entre outros (LOUZADA et al, 2015; MASCARENHAS, et al, 2014; OTONNI; CARLOS, 2012).

A atividade de dirigir é desgastante, causa fadiga e sua eficácia está relacionada, principalmente, a fatores ambientais, físicos e psíquicos que afetam não só a atividade de dirigir mas também a vida social e coletiva desse profissional, provocando riscos à saúde (SANTOS JUNIOR, 2002; RAMOS; ALMEIDA; RIBEIRO, 2018).

A diabetes também foi referida neste estudo por 53,0\% dos entrevistados e está relacionada, ainda, com as doenças gastrointestinais citadas por $23,5 \%$ dos participantes. Um estudo internacional mostrou a associação entre a DM, a obesidade e fatores ambientais. Em um projeto que avaliou a população de crianças e adolescentes com DM1 na Alemanha, Áustria e Estados Unidos, os pacientes apresentaram maior Índice de Massa Corporal-IMC em relação aos valores de referência: $12 \%$ deles tinham obesidade e $24 \%$ se encontravam com excesso de peso (CHOBO; GÓROWSKA - KOWOLIK; SOKOŁOWSKA; JAROSZ - CHOBOT, 2018).

As doenças gastrointestinais são bastante comuns na população. Essas são influenciadas por múltiplos fatores como genéticos, ambientais, imunológicos e nutricionais. O aumento da incidência dessas enfermidades tem sido associado à industrialização à 
ocidentalização no estilo de vida, incluindo hábitos alimentares e tabagismo. Os principais sintomas são diarréia, dor abdominal, sangramento gastrointestinal, perda de peso, subnutrição e fadiga (LOUZADA et al, 2015; OLIVEIRA, et al, 2018; GIROTTO; LOCH, MESAS, GONZÁLEZ; GUIDONI; ANDRADE, 2020).

A atividade e o ambiente em que os motoristas executam seus trabalhos os colocam em um grupo de risco para determinados problemas de saúde, apresentando prevalências preocupantes de fatores de risco para doenças crônicas (MOURA NETO; SILVA, 2013; FERREIRA; GRAMS; ERTHAL; GIRIANELLI; OLIVEIRA, 2018).

Estudos têm mostrado que alguns parâmetros de qualidade de vida hábitos e estilo de vida, sedentarismo, alimentação inadequada, poucas horas de sono, tabagismo, estresse, etc. - interferem na exposição dos motoristas profissionais aos riscos cardiovasculares e que são maiores do que na população em geral, mas também, de outras doenças (ALCANTARA et al, 2016; CARDOSO, 2015; SILVEIRA; ABREU; SANTOS, 2014; FERREIRA; ALVAREZ, 2013; MOURA NETO; SILVA, 2012).

A esse respeito é bastante elucidativo um estudo realizado com 400 caminhoneiros do interior de São Paulo, na Rodovia SP-330 com o objetivo de analisar aspectos de saúde e de trabalho e identificar possíveis relações, determinantes e/ou agravantes, de impactos negativos na sua qualidade de vida. Os autores utilizaram um questionário fechado, que abordava aspectos da percepção dos motoristas sobre a sua saúde e trabalho. Dentre os entrevistados $67,75 \%$ referiram problemas constantes ou ocasionais de postura; $37,75 \%$, auditivos; $57,5 \%$ estomacais; $58,5 \%$ sentimentos negativos como medo, estresse e depressão. Quanto aos hábitos de consumo e comportamentos $87,75 \%$ ingeriam café; $84,5 \%$ alimentos gordurosos; $43 \%$ bebidas alcoólicas; $19,5 \%$ energéticos; $32 \%$ fumavam e $2 \%$ usavam outras drogas. Diversos aspectos das condições de trabalho, hábitos e comportamentos exercem impactos negativos sobre sua saúde, com prejuízos para a qualidade de vida (PENTEADO; GONÇALVES; COSTA; MARQUES, 2008).

Por último foi indagado o que poderia ser feito para melhorar as condições de vida desse grupo. A maior parte respondeu $(78,0 \%)$ que a cooperativa poderia ser mais comprometida com os associados.

\footnotetext{
"A cooperativa não oferece serviços para os seus cooperados". "Não temos apoio para o trabalho".

"A cooperativa cobra uma taxa, mas não oferece nada em troca".

"Não há atividades de lazer para os associados nem para a família".
} 
"Poderia ter um plano de saúde para os membros".

"A cooperativa cobra uma mensalidade, mas não oferece contrapartida".

"Os colegas são muito competitivos".

Constatou-se, durante as entrevistas, que as queixas são pertinentes. No entanto, os motoristas, colocam a responsabilidade da resolução desses problemas na direção da cooperativa. Esquecem que esta é feita pelos seus associados. A ideia do que é e como funciona uma cooperativa não parece ser muito bem entendida pelos participantes.

\section{Considerações finais}

Os dados coletados através das entrevistas, permitiram mostrar que os problemas de saúde mais prevalentes entre os motoristas são aqueles relacionados com a atividade laboral desenvolvida por esses profissionais e com os estilos de vida que afetam as condições de vida e de trabalho.

Os resultados obtidos não permitem generalizações uma vez que o número de entrevistados foi pequeno. No entanto, é possível que essa realidade seja encontrada entre outros motoristas que estejam submetidos a semelhantes condições de trabalho, saúde e vida. O estudo mostrou resultados parecidos com outros realizados com motoristas em outras regiões do Brasil e em outros países o que leva a inferir que a realidade de trabalho desse grupo é precária e independente da região e do país.

Além disso, o trabalho do motorista pode levar a prejuízos à saúde, por favorecer a adoção de estilos de vida pouco saudáveis como sedentarismo, inadequados hábitos alimentares, sobrepeso, uso de álcool e tabaco, entre outros. A profissão também interfere na convivência familiar e vida social do motorista, pois, o afasta de sua família, amigos, datas importantes. As condições de vida e trabalho nem sempre são favoráveis à manutenção da saúde. Os fatores de risco mais citados foram aqueles relacionados com o ambiente físico.

A prevalência de doenças crônicas como a hipertensão e a diabetes além das osteomusculares pode estar associada a comportamentos preventivos e estilos de vida pouco saudáveis. As equipes de saúde que atendem esse grupo de profissionais devem destacar a importância dos programas de educação em saúde, encorajando a adoção e manutenção de padrões de vida sadios e estimulando a tomada de decisões preventivas que modifiquem comportamentos de risco. Portanto, é necessário um conjunto de intervenções 
interdisciplinares relacionadas que levem em consideração as necessidades de conforto físico e psíquico dos motoristas. Há, ainda, riscos psicossociais na atividade desenvolvida pelos participantes do estudo o que repercute de forma direta na qualidade de vida e nas condições de trabalho. Um serviço de apoio aos trabalhadores deve, entre outros quesitos, responder às necessidades individuais do grupo.

Assim sendo, devem-se investir na melhoria da saúde desses profissionais, promovendo um ambiente de trabalho, onde ações de prevenção e de promoção à saúde sejam realizadas a fim de minimizar o impacto desses fatores.

É responsabilidade de todos buscarem novas formas de lidar com o trabalho, para que os envolvidos melhorem as suas condições de trabalho. Devem ser informados acerca dos riscos a que estão expostos, oferecendo-lhes suporte social e psicológico,

Face aos aspectos evidenciados no estudo, devem ser estimuladas mudanças quanto aos hábitos e estilos de vida, a partir da realização de atividades físicas regulares, controle do peso corporal, diminuição da ingestão de bebidas alcoólicas e abstinência do tabagismo, entre outras.

Assim sendo, espera-se que esses resultados possam servir de subsídios para o planejamento de programas voltados para prevenção, promoção da saúde e controle dos agravos, a fim de melhorar as condições de saúde desses profissionais. Sugerem-se estímulo e suporte para a realização de novas pesquisas na área.

\section{Agradecimentos}

Ao CNPq - Conselho Nacional de Desenvolvimento Científico e Tecnológico, pela concessão da bolsa PIBIC no período de 2014 a 2016, possibilitando, assim, apoio financeiro para a realização da pesquisa.

\section{Referências}

ALCANTARA, V.C.G. et al. A qualidade de vida para motoristas de ônibus: entre a saúde e o trabalho. Revista Portuguesa de Enfermagem de Saúde Mental. Porto, n. spe4, p. 101-106, out. 2016.

ÁLVARES, J et al. Pesquisa Nacional sobre Acesso, Utilização e Promoção do Uso Racional de Medicamentos: métodos. Rev. Saúde Pública. v. 51 (suppl 2), n. 13, p. 1-9, 2017. 
ANTUNES, R. Adeus ao trabalho? Ensaio sobre as metamorfoses e a centralidade do mundo do trabalho. 15 ed. São Paulo: Cortez, 2013.

ASSUNÇÃO, A.A; FRANÇA, E.B. Anos de vida perdidos pelo CNCD atribuídos a riscos ocupacionais no Brasil: estudo GBD 2016. Rev. Saúde Pública. São Paulo v.54, n. 28, p. 1-12, 2020 .

BRASIL. Constituição da República Federativa do Brasil. São Paulo: Saraiva, 2018.

BRASIL. Lei 12.619, de 30 de abril de 2012. Dispõe sobre o exercício da profissão de motorista, Brasília. Disponível em: www.planalto.gov.br. Acesso em 10 de ago de 2016

BRITO, G. M.; GOIS, C. F. L.; ALMEIDA, A. F.; MARTINS, A. W. R.; RODRIGUES, E.O.L.; GOIS JUNIOR, J. P. Fatores de risco para hipertensão arterial entre motoristas de ônibus. Revista Baiana de Enfermagem, Salvador, v. 30, n. 2, p. 1-7, abr./jun. 2016.

CAMARGO, E. P. et al. A população está devidamente informada sobre os distúrbios do sono? Arq. Neuro-Psiquiatr. 2013, v.71, n.2, p.92-99.

CARDOSO, A.C.; MORGADO, L. Trabalho e saúde do trabalhador no contexto atual: ensinamentos da Enquete Europeia sobre Condições de Trabalho. Saude Soc São Paulo. v.28 n.1. p. 169-181, 2019.

CARDOSO, A.C.M. O trabalho como determinante do processo saúde-doença. Tempo soc. São Paulo, v.27 n.1 p. 73-94, 2015.

CHOBO, A; GÓROWSKA - KOWOLIK, K; SOKOŁOWSKA, M; JAROSZ - CHOBOT, P. Obesyt and Diabets - not only a link simple betwen two epidemics. Diabetes Met Res Rev. v. 34. n. 7, p.19, 2018.

DE VITTA, A; CONTI, M.H.S; TRIZE, D.B; QUINTINO, N.M; PALMA, R; SIMEÃO, S.F.A..P. Sintomas musculoesqueléticos em motoristas de ônibus: prevalência e fatores associados. Fisioter. mov. Curitiba v.26 n.4, p.863-71, 2013.

FERREIRA, A.P.; GRAMS, M.T., ERTHAL, R.M.C, GMHSIRIANELLI, V.R, OLIVEIRA M.H.B. Revisão da literatura sobre os riscos do ambiente de trabalho quanto às condições laborais e o impacto na saúde do trabalhador. Rev Bras Med Trab. v.16, n.3, p.360-370, 2018.

FERREIRA, S.S; ALVAREZ, D. Organização do trabalho e comprometimento da saúde: um estudo em caminhoneiros. Sistemas \& Gestão, n.8, 2013, pp 58-66.

GIROTTO, E.D; LOCH, M.R; MESAS, A.E; GONZÁLEZ, A.D; GUIDONI, C.M; ANDRADE, S.M. Comportamentos alimentares de risco à saúde e fatores associados entre motoristas de caminhão. Ciênc. Saúde Colet. v. 25, n. 3, p. 1011-1023, 2020.

KUBOTA, A. et al. Aspectos da insônia no adulto e a relação com o desempenho ocupacional. Rev Ter Ocup Univ São Paulo. v. 25, n.2, p. 119-25, 2014. 
LOUZADA, MLC et al. Alimentos ultraprocessados e perfil nutricional da dieta no Brasil. Rev Saúde Pública. v. 49, n.38, p. 1-11, 2015.

MASCARENHAS, C.H.M et al. Prevalência de dor lombar em Motoristas de táxi do município de Jequié-Ba. Revista Espaço para a Saúde. Londrina, v. 15, n. 1, p. 66-76, 2014

MASSON, V.A; MONTEIRO, M.I. Estilo de vida, aspectos de saúde e trabalho de motoristas de caminhão Estilo de vida, aspectos de saúde e trabalho de motoristas de caminhão. Rev Bras Enferm. Brasília, v.63, n.4, p. 533-40, 2010.

MORAES, TD; SANTORUM, K; SOUZA, FVB; ÁVILA, LR; VIEIRA, SS. Considerações sobre o ofício de dirigir ônibus no Brasil: uma revisão de literatura. Est. Inter. Psicol. Londrina, v.8 n.1, jan.-jun. 2017.

MOURA NETO, A,B; SILVA, M,C. Diagnóstico das condições de trabalho, saúde e indicadores do estilo de vida de trabalhadores do transporte coletivo da cidade de Pelotas - RS. Rev Bras Ativ Fis e Saúde. Pelotas/RS. v.17, n.5, p. 347-358. 2012.

NEFFA, J,C. O trabalho humano e a sua centralidade. Revista Ciências do Trabalho, n. 4, p. 726, 2015.

NEVES, G,S,M,L; MACEDO, P; GOMES, M,M. Transtornos do sono: atualização (1/2.). Rev Bras Neurol. v.53 n.3, p. 19-30, 2017.

OTTANI, K.P; CARLOS, C,M,G. Motoristas profissionais no trânsito e suas consequências. UNAR Araras (SP), v. 6, n. 1, p. 61-72, 2012. ISSN 1982-4920).

PENTEADO, R.Z; GONÇALVES, C.G.O; COSTA, D.D; MARQUES, J.M. Trabalho e saúde em motoristas de caminhão no interior de São Paulo. Saude soc. São Paulo, v.17 n.4, p. 3545, out./dez. 2008.

RAMOS, B.H.; ALMEIDA, M.F.C.; RIBEIRO, J.A.; SILVEIRA, C.A. Condições de vida, trabalho e saúde de motoristas de transporte de cargas. Rev enferm UFPE on line. Recife, v.12, n. 1, p.150-9, 2018.

SANTOS JÚNIOR, E.A. De que adoecem e morrem os motoristas de ônibus? Uma revisão da literatura. Rev. Bras. Med. Trab. Belo Horizonte. v, 1 n.2 p. 138-147, 2003.

SANTOS, A.M.S; RODRIGUES, B.B; VENANCIO, J.C; BRAGANÇA E SILVA, G; FERREIRA, GCO; FERNANDES, TM. Uso de drogas por caminhoneiros que trafegam na rodovia BR-153. Rev. méd. Minas Gerais, v. 27, n. 1-4, p. 27-30, 2017. DOI: http://dx.doi.org/10.5935/22383182.20170052

SILVA M.S; ASSUMPÇÃO L.O.T; NEVES, R.L.R. Avaliação da qualidade de vida e saúde de caminhoneiros de Gurupi - TO. Revista Brasileira de Ciências da Saúde, ano 11, n. 35, p. 1624, 2013 
SILVA, L.G; LUZ, A.A; VASCONCELOS, S.P; MARQUEZE, E.C; MORENO, C.R.C. Vínculos empregatícios, condições de trabalho e saúde entre motoristas de caminhão Revista Psicologia: Organizações e Trabalho, v. 16, n. 2, p. 153-165, 2016.

SILVEIRA, L.S; ABREU, C.C; SANTOS, E.M. Análise da Situação de Trabalho de Motoristas em uma Empresa de Ônibus Urbano da Cidade de Natal/RN. PSICOLOGIA: CIÊNCIA E PROFISSÃO, v. 34, n.1, p.158-179, 2014.

SOUZA, L.P.S; SILVA, JJ; SILVA, C.S.O; PINTO, I.S. Prevalência e fatores associados à hipertensão em trabalhadores do transporte coletivo urbano no Brasil. Rev. bras. med. Trab, v. 15, n.1, p. 80-87, 2017.

TORRES, J.C.R. Oitava Conferência Nacional de Saúde: o SUS ganha forma. EPSJV/Fiocruz. Disponível em: http://www.epsjv.fiocruz.br. Acesso em: 29.06.2020 